\title{
Mediastinal germ cell tumour masquerading as loculated pleural effusion
}

\author{
Gopal Chawla', Pranav Ish², Avneet Garg ${ }^{3}$, Kunal Deokar ${ }^{4}$ \\ 'All India Institute of Medical Sciences, Jodhpur, Basni , Jodhpur, India \\ ${ }^{2}$ Vardhaman Mahavir Medical College and Safdarjung Hospital, Delhi, India \\ ${ }^{3}$ All India Institute of Medical Sciences, Delhi, India \\ ${ }^{4}$ All India Institute of Medical Sciences, Jodhpur, Basni , Jodhpur, India
}

We report a case of a large teratoma arising from the anterior mediastinum that presented as a confusing clinical picture of loculated pleural effusion which was successfully diagnosed and treated by surgical excision.

A 17-year-old girl with no comorbid conditions presented with trivial breathlessness and occasional cough. She was being treated for upper respiratory tract infection on and off for the past 6 months. Her breathlessness was greater while she played basketball at school and was associated with a cough which was dry in nature. She denied any fever, weight loss, previous disease, or any menstrual irregularities. Her physical examination revealed that she was apyrexial, normotensive, and eupnoeic. Her thyroid, lymph node, abdominal, and per-vaginal examinations were normal apart from decreased breath sounds in her right side. A chest X-ray revealed right-sided homogenous opacity with regular margins widening the cardiac shadow. There was no previous radiology for comparison. The suspicion of massive loculated effusion or some congenital cyst was made. USG of the chest showed the anechoic area with septations with isoechoic area making the possibility of loculated effusion or empyema more likely. Considering the patient's trivial symptoms and non-toxic nature which would have not been there in case of empyema, contrast-enhanced computerised tomogram was done. CT scan showed a large well-defined mass, which was low attenuating with focal areas of fat and calcification along with areas of cystic degeneration. Trucut biopsy was suggestive of mature cystic teratoma. The patient underwent open thoracotomy and enucleation of the tumour. Surgical and pathologic findings led to the diagnosis of a mature cystic teratoma with components of three germ layers. Gross examination showed a rounded tumour measuring $18 \times 11.5 \times 7.8 \mathrm{~cm}$ and weighing $300 \mathrm{~g}$. The tumour was predominantly cystic, with thin, sharply delineated wall filled with sebaceous material and hair which mimicked loculated septated effusion on USG. Microscopically, the cyst wall was lined by the epidermis and some are lined by tall columnar with underlying sebaceous glands and hair follicles. The walls of the cyst showed pilosebaceous units and eccrine glands lobules of mature adipose tissue, hyaline cartilage, brain tissue and nerve twigs (Figure 1).

Germ cell tumours are the fourth most common mediastinal neoplasm, occurring almost always in the anterosuperior compartment. In this location, germ cell tumours are second only to thymomas and lymphomas. Five percent of germ cell tumours are extragonadal, the anterior mediastinum is the most common location followed by the retroperitoneum, pineal and suprasellar regions; and men are affected more often than women [1]. Microscopically, they are characterised by well-differentiated derivations from at least two of the three germ cell layers (ectoderm, mesoderm, and endoderm). Ectodermal elements may be represented by skin, teeth, and hair; mesodermal elements by bone, cartilage, and muscle; and endodermal elements by bronchial and gastrointestinal epithelium and pancreatic tissue. Cyst formation is typical, and at times they have raised protuberance projecting into the cyst cavity known as Rokitansky Nodule [2].

Most mediastinal teratomas like our case are asymptomatic or have trivial complaints unless they compress adjacent structures like the airways and vessels. Dull aching chest pain is often the most common presentation. At times some patients present with coughing up hair, i.e. trichoptysis indicating communication between the tracheobronchial tree and the teratoma. Physical examination is limited to chest dullness and diminished breath sounds. While some individuals have a bulging deformity of the chest wall, a draining fistula between the tumour and the skin, acanthosis nigricans, or superior vena cava obstruction [3].

Radiologically, teratomas are round or lobulated anterior mediastinal mass that has sharp margins and occupies one side of the thorax. Calcification, ossification, or even teeth may even be visible on chest radiographs. Chest CECT is the imaging modality of choice. Mediastinal mature teratomas contain soft tissue in virtually all cases followed by fluid in $88 \%$ and fat in $76 \%$. All these elements are present in the same lesion

Address for correspondence: Gopal Chawla, All India Institute of Medical Sciences, Jodhpur, Basni , Jodhpur, India; e-mail: dr.gopalchawla@gmail.com DOI: 10.5603/ARM.a2020.0116

Received: 09.02.2020

Copyright (C) 2020 PTChP

ISSN 2451-4934

Conflict of interest: None declared 

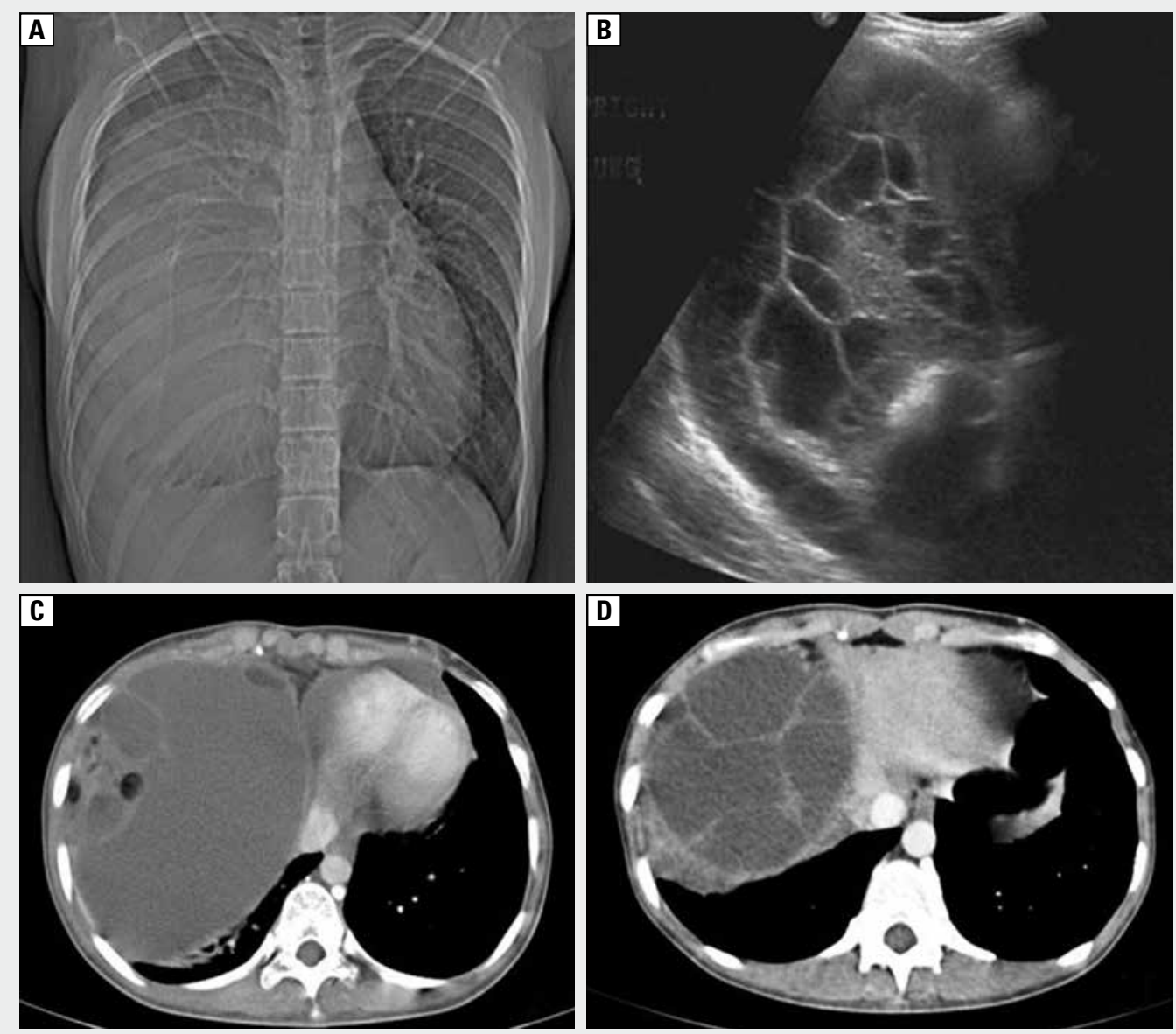

Figure 1. A. Chest X-ray showing homogenous opacity at the right side; B. USG of the chest showing effusion with multiple loculations; C. Chest CT showing large well-defined mass, which is low attenuating with focal areas of fat and calcification, areas of cystic degeneration; D. Anterior mediastinal cystic mass with solid enhancing septa

in $39 \%$ of cases. In $15 \%$ of patients, teratomas consist only of cystic lesions that contain neither fat nor calcification. A fat-fluid level within the mass is a highly specific finding but is seen less frequently. Teratoma may rupture at times, which radiologically is seen as inhomogeneity of the internal component of the mass or adjacent consolidation or pleural or pericardial effusion [4-5].

\section{References:}

1. Duwe B, Sterman D, Musani A. Tumors of the mediastinum. Chest. 2005; 128(4): 2893-2909, doi: 10.1378/chest.128.4.2893.

2. Rosado-de-Christenson ML, Templeton PA, Moran CA. From the archives of the AFIP. Mediastinal germ cell tumors: radiologic and pathologic correlation. Radiographics. 1992; 12(5): 1013-1030, doi: 10.1148/radiographics.12.5.1326777, indexed in Pubmed: 1326777.

3. Yalagachin GH. Anterior mediastinal teratoma - a case report with review of literature. Indian J Surg. 2013; 75(Suppl 1): 182-184, doi: 10.1007/s12262-012-0569-6, indexed in Pubmed: 24426558.

4. Moeller KH, Rosado-de-Christenson ML, Templeton PA. Mediastinal mature teratoma: imaging features. AJR Am J Roentgenol. 1997; 169(4): 985-990, doi: 10.2214/air.169.4.9308448, indexed in Pubmed: 9308448.

5. Fulcher AS, Proto AV, Jolles H. Cystic teratoma of the mediastinum: demonstration of fat/fluid level. AJR Am J Roentgenol. 1990; 154(2): 259-260, doi: 10.2214/ajr.154.2.2105009, indexed in Pubmed: 2105009. 